ISSN 0103-5150

Fisioter. Mov., Curitiba, v. 24, n. 2, p. 231-238, abr./jun. 2011

Licenciado sob uma Licença Creative Commons

\title{
Alteração espirométrica em crianças com mielomeningocele é dependente do nível de lesão funcional
}

\author{
Spirometric abnormalities in children with myelomeningocele is \\ dependent of the level of functional injury
}

\author{
Renata Calhes Franco de Moura ${ }^{[a]}$, Flavia Miranda ${ }^{[b]}$, Leandra Marques de Souza ${ }^{[c]}$, \\ Simone Dal Corso ${ }^{[\mathrm{d}]}$, Carla Malaguti ${ }^{[\mathrm{e}]}$
}

[a] Mestre em Distúrbios do Desenvolvimento, professora do curso de Fisioterapia da Universidade Nove de Julho (Uninove), São Paulo, SP - Brasil, e-mail: renatacalhes@uninove.br

[b] Graduanda do curso de Fisioterapia da Universidade Nove de Julho (Uninove), São Paulo, SP - Brasil, e-mail: flaviamartinsf@hotmail.com

[c] Mestranda em Ciências da Reabilitação da Universidade Nove de Julho (Uninove), São Paulo, SP - Brasil, e-mail: souzaleandra23@yahoo.com.br

[d] Doutora em Ciências, professora do Mestrado em Ciências da Reabilitação da Universidade Nove de Julho (Uninove), São Paulo, SP - Brasil, e-mail: simonedc@uninove.br

[e] Doutora em Ciências, professora do Mestrado em Ciências da Reabilitação da Universidade Nove de Julho (Uninove), São Paulo, SP - Brasil, e-mail: carlamalaguti@uninove.br

\section{Resumo}

Objetivo: Avaliar a função pulmonar de crianças com mielomeningocele (MMC) de diferentes níveis de lesão funcional comparados a controles saudáveis pareados por idade e sexo. Material e método: Este foi um estudo caso-controle, no qual foi selecionada apenas uma criança por nível funcional de lesão e com função cognitiva preservada, e controles de crianças saudáveis pareados por idade e gênero ao grupo de MMC. Medidas antropométricas e espirométricas de função pulmonar foram obtidas de ambos os casos e controles de MMC. Resultados: Todas as crianças com MMC apresentaram reduzido estado nutricional segundo os índices recomendados pela OMS. Foi observada presença de distúrbio ventilatório restritivo leve nas crianças com nível de lesão torácica ( $\mathrm{CVF}=0,65 \%$ prev) e lombar-alta (CVF = 0,69 \%prev), e apesar de dentro dos limites da normalidade, as crianças com MMC com níveis inferiores de lesão apresentaram valores espirométricos menores do que seus respectivos controles. Houve correlação negativa perfeita entre a capacidade vital forçada e o nível de lesão funcional das crianças com MMC. Conclusão: Crianças com MMC apresentam reduzidos valores de função pulmonar quando comparados aos controles 
saudáveis, sobretudo os com níveis de lesão funcional elevada, os quais demonstraram distúrbio ventilatório restritivo. A fisioterapia respiratória deve ser incorporada na avaliação e segmento das crianças com MMC, coadjuvante à fisioterapia motora, especialmente naquelas acometidas com níveis de lesões mais elevadas.

Palavras-chave: Mielomeningocele. Métodos de Avaliação. Espirometria. Manifestações neuromusculares. Terapia respiratória.

\begin{abstract}
Objective: To evaluate pulmonary function in children with myelomeningocele (MMC) of different functional levels of injuries. Study design: It was a case-control study, which enrolled one child with myelomeningocele by level of injury and control of health children matched for age and sex. Methods: Anthropometric and spirometric measures were obtained from both groups. Results: All of children with MMC showed reduced nutritional state according OMS parameters. We observed the presence of mild restrictive ventilatory disturbance in the children with injuries on thoracic (FVC $=0.65 \%$ pred) and lumbar-high level (forced vital capacity $-F V C=0.69 \%$ pred), and although with normal limits, children with MMC with lower levels of injury presented lower spirometric values than their respective controls. There was a perfect negative correlation between forced vital capacity and level of functional lesions. Conclusions: Children with MMC presented reduced lung function values when compared to healthy controls, especially those with high levels of functional injury, which showed restrictive ventilatory disturbance. Respiratory therapy should be incorporated in the assessment and in the follow up of children with MMC, as adjunct to neuromotor therapy, especially those with higher levels of injury.
\end{abstract}

Keywords: Myelomeningocele. Assessment methods. Spirometry. Neuromuscular manifestations. Respiratory therapy.

\section{Introdução}

A mielomeningocele (MMC) é uma malformação que ocorre até a quarta semana de embriogênese, responsável por cerca de $85 \%$ dos distúrbios do fechamento do tubo neural (DFTN) presentes ao nascimento, caracterizando-se por falha no fechamento dos arcos vertebrais posteriores, levando à exposição do tecido nervoso, que resulta em crescimento displásico de estruturas do Sistema Nervoso Central, formando uma bolsa cística ao nível da lesão $(1,2)$.

Diversas manifestações são encontradas, como: disfunção cardíaca, displasia renal, hiperplasia adrenal e alterações que estão diretamente relacionadas ao nível da lesão neurológica, como: hidrocefalia, alteração cognitiva, bexiga neurogênica, paralisia de membros inferiores, deformidades posturais e distúrbios respiratórios do sono (3-7). Todos estes aspectos deterioram com a idade, especialmente aqueles com nível de lesão mais alta. Atribuem-se como principais causas de morte as complicações pulmonares, cardiovasculares e renais. Diante da alta probabilidade dos pacientes com MMC se tornarem cadeirantes, estando a MMC associada à imobilidade e ao agravamento da cifoescoliose, pode ocorrer baixa reserva ventilatória, aumentando os riscos de complicações pulmonares $(8,9)$. Além disso, estudos observaram que crianças com mielomeningocele nas idades estudadas apresentaram redução da força muscular respiratória mais significante na lesão medular alta $(10,11)$.

Embora a literatura científica tenha amplamente estudado as manifestações clínicas dos diferentes níveis da MMC, pouca atenção tem sido dada à disfunção pulmonar resultante das anormalidades neuromusculares e ortopédicas que se manifestam nesta doença (12-15).

Neste sentido, este estudo objetivou avaliar a função pulmonar em crianças com mielomeningocele de diferentes níveis de lesão funcional, quando comparadas a controles saudáveis pareados por idade e sexo. 


\section{Materiais e métodos}

\section{Amostra}

Este foi um estudo caso-controle, no qual a amostra de conveniência de pacientes portadores de MMC foi recrutada de uma clínica-escola de fisioterapia, enquanto o grupo controle de crianças saudáveis foi recrutado de uma clínica-escola de odonto-pediatria. Este estudo foi aprovado pelo Comitê de Ética em Pesquisa institucional (Processo n. 263235). 0 consentimento livre e esclarecido dos pais ou responsáveis de todas as crianças foi obtido antes do início do protocolo de estudo.

Foram incluídas no estudo crianças com idade de 4 a 12 anos com diagnóstico de MMC, e foi selecionado apenas um paciente por nível funcional de lesão e com função cognitiva preservada. 0 grupo controle de crianças saudáveis foi incluído pareado por idade e gênero ao grupo de MMC. Foram excluídas do estudo crianças que não colaboraram ou não conseguiram realizar a manobra espirométrica a contento, as com história de asma pregressa ou outras doenças respiratórias e/ou aquelas com deformidades posturais nas crianças do grupo controle.

\section{Avaliação antropométrica}

Para a obtenção das medidas antropométricas de peso, foi utilizada uma balança calibrada da marca Welmy ${ }^{\circledR}$ (São Paulo, Brasil) com plataforma e capacidade de até $150 \mathrm{~kg}$. Para a tomada da estatura foi utilizado o estadiômetro da balança (certificada pelo Inmetro) com limites entre $1 \mathrm{~m}$ e $2 \mathrm{~m}$. Baixo peso, obesidade e o eutrofismo foram determinados por meio do índice de massa corporal (IMC), calculado pela equação: $\mathrm{IMC}=$ peso $(\mathrm{kg}) /$ estatura $^{2}(\mathrm{~m})$. Foi ainda preconizado o valor de percentil maior que $90 \%$ para crianças obesas, entre $10 \%$ e $90 \%$ para crianças eutróficas e abaixo de $10 \%$ como crianças de baixo peso. A fonte gráfica para a determinação dos limites de percentil utilizados foram os mesmos recomendados pela Organização Mundial de Saúde (16).

\section{Espirometria}

Para a realização da espirometria foi utilizado o espirômetro portátil da Cosmed $\mathrm{Srl}^{\circledR}$ modelo Pony
Spirometer Graphic (Roma, Itália). A manobra para a realização da espirometria consiste em uma inspiração profunda, seguida de manobra expiratória forçada, mantida até que o indivíduo não a tolere mais, ou até que sejam atingidos os critérios de aceitação propostos pelas Diretrizes para Testes de Função Pulmonar da Sociedade Brasileira de Pneumologia e Tisiologia (17). Todas as crianças realizaram no mínimo três manobras de capacidade vital forçada. A partir destas manobras foram obtidos os gráficos fluxo-volume e volume-tempo, além do valor numérico das seguintes variáveis: capacidade vital forçada (CVF), volume expiratório forçado no primeiro segundo $\left(\mathrm{VEF}_{1}\right)$ e relação $\mathrm{VEF}_{1} / \mathrm{CVF}$. Os valores previstos foram obtidos a partir dos valores de referência para crianças e adolescentes descritos por Polgar e Promadhat (18).

\section{Análise estatística}

Os dados individuais antropométricos e espirométricos estão apresentados de forma descritiva. Coeficiente de correlação de Spearman foi usado para verificar o nível de associação entre as variáveis de função pulmonar e nível de lesão funcional nos portadores de MMC. Foi assumido como significância estatística o valor de $\mathrm{p} \leq 0,05$.

\section{Resultados}

Quatro crianças com MMC (2F, $2 \mathrm{M})$, com diferentes níveis de lesões funcionais (sacral, lombar baixo, lombar alto e torácica), e quatro crianças saudáveis foram avaliadas. As características antropométricas estão apresentadas na Tabela 1, em que se observam menores valores de IMC e curva de percentil no grupo de crianças com MMC, comparado aos controles saudáveis. Na Tabela 2 pode-se observar que os valores espirométricos das crianças com MMC com níveis de lesão funcional torácico (cadeirante) e lombar alto foram compatíveis com distúrbio ventilatório restritivo leve, enquanto as com níveis de lesão lombar baixa e sacral, embora inferiores aos seus respectivos controles (Figura 1), estiveram nos limites da normalidade. Por outro lado, como esperado, os valores espirométricos basais das crianças do grupo controle estiveram dentro dos valores de normalidade. Nenhum distúrbio ventilatório do tipo obstrutivo foi observado nas crianças com MMC. 
de Moura RCF, Miranda F, de Souza LM, Dal Corso S, Malaguti C.

Tabela 1 - Características antropométricas dos grupos de MMC e controle

\begin{tabular}{lcccccc}
\hline Nível funcional de lesão (MMC) & IMC $\left(\mathrm{Kg} / \mathbf{m}^{2}\right)$ & Curva de percentil (\%) & Idade/Sexo & \multicolumn{1}{c}{$\mathbf{I M C}\left(\mathrm{Kg} / \mathrm{m}^{2}\right)$} & Curva de percentil (\%) \\
\hline & Grupo $\mathbf{M M C}$ & & & & \multicolumn{2}{c}{ Grupo Controle } \\
\hline Torácico & 13,5 & 3 & $11 / \mathrm{F}$ & & 18,3 & 75 \\
Lombar alto & 14,0 & 3 & $5 / \mathrm{F}$ & & 17,8 & 91 \\
Lombar baixo & 15,5 & 3 & $12 / \mathrm{M}$ & & 20,7 & 50 \\
Sacral & 13,6 & 3 & $4 / \mathrm{M}$ & & 17,2 & 75 \\
\hline
\end{tabular}

Tabela 2 - Características espirométricas dos grupos de MMC e controle

\begin{tabular}{|c|c|c|c|c|c|c|c|}
\hline Nível de lesão & CVF (L, \%p) & $\operatorname{VEF}_{1}(\mathrm{~L}, \% p)$ & $\mathrm{VEF}_{1} / \mathrm{CVF}$ & Idade/Sexo & CVF (L, \%p) & $\operatorname{VEF}_{1}(\mathrm{~L}, \% \mathrm{p})$ & $\operatorname{VEF}_{1} / \mathrm{CVF}$ \\
\hline \multicolumn{5}{|c|}{ Grupo MMC } & \multicolumn{3}{|c|}{ Grupo Controle } \\
\hline Torácico & $1,47(0,65)^{\star}$ & $1,44(0,67)$ & 0,98 & $11 / \mathrm{F}$ & $1,12(0,90)$ & $1,08(0,95)$ & 0,96 \\
\hline Lombar alto & $0,69(0,69)^{*}$ & $0,69(0,76)$ & 0,99 & $5 / F$ & $0,98(0,95)$ & $0,91(0,93)$ & 0,98 \\
\hline Lombar baixo & $2,19(0,78)$ & $2,04(0,80)$ & 0,93 & $12 / \mathrm{M}$ & $2,81(1,00)$ & $2,51(1,07)$ & 0,89 \\
\hline Sacral & $0,95(0,82)$ & $0,75(0,74)$ & 0,79 & 4/M & $1,16(1,06)$ & $1,12(1,03)$ & 0,96 \\
\hline
\end{tabular}

Legenda: * = presença de distúrbio ventilatório restritivo leve.

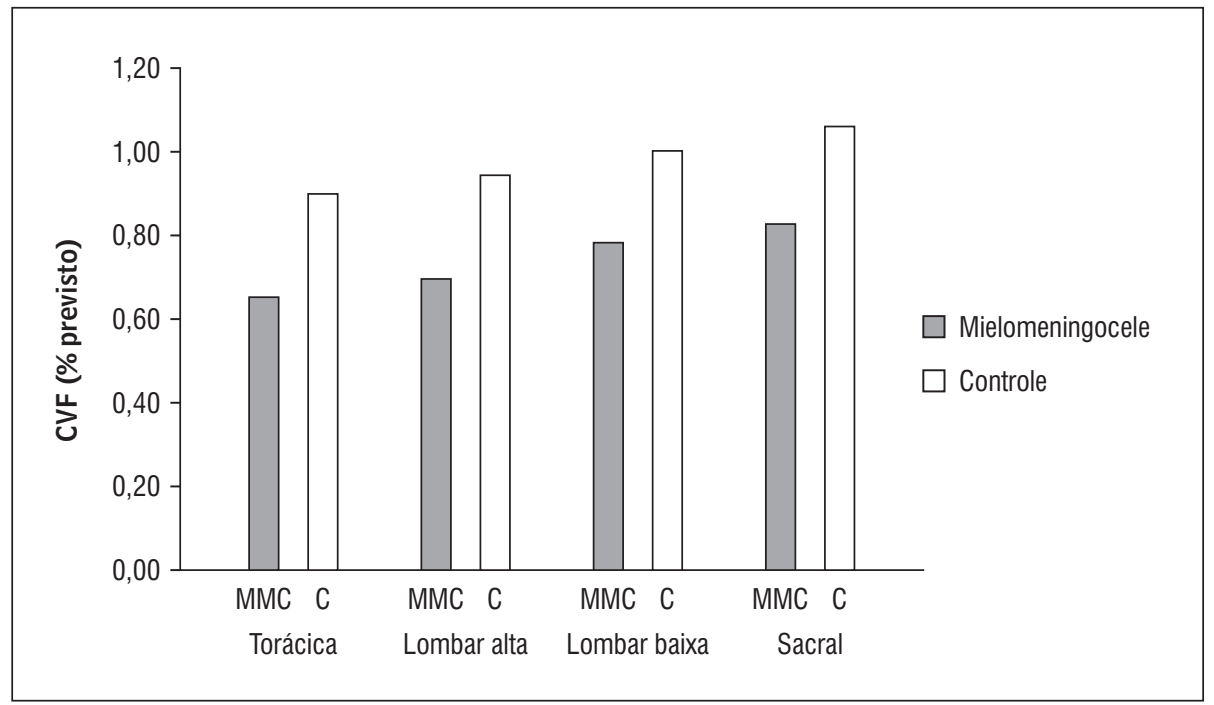

Figura 1 - Representação gráfica dos valores de capacidade vital forçada dos casos de MMC com diferentes níveis de lesões e seus respectivos controles

Quando comparados os valores espirométricos em termos absolutos, entre os casos e controles, podemos observar que as crianças com MMC apresentaram valores espirométricos inferiores aos controles, com exceção apenas da criança com nível de lesão torácica, que, embora tenha apresentado valores absolutos superiores ao controle de mesma idade e gênero, apresentou-se ainda com valores previstos inferiores ao da normalidade.

Pode se observar na Figura 2 associação negativa perfeita $(r=-1,00 ; p \leq 0,001)$ entre o indicador de restrição pulmonar (CVF - \% do previsto) e o nível de lesão funcional nas crianças com MMC. 


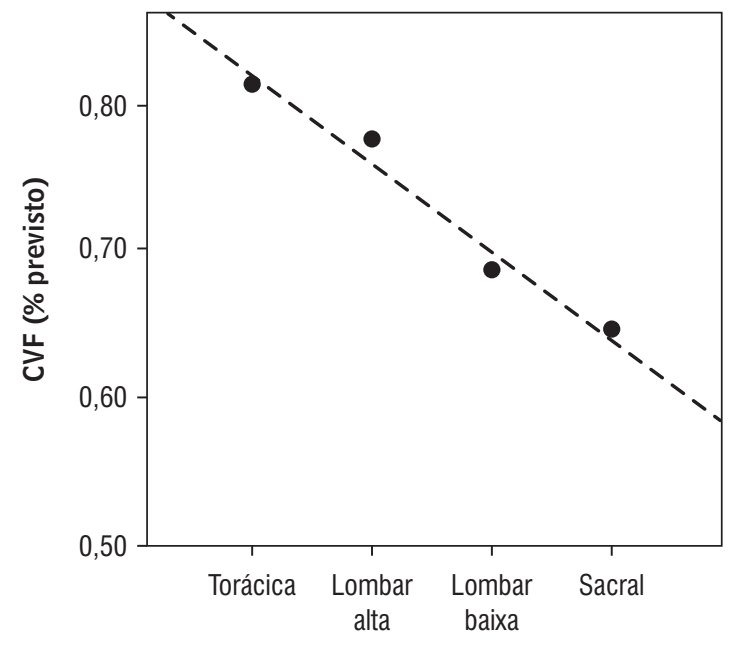

Figura 2 - Relação entre o nível de lesão funcional e capacidade vital forçada das crianças portadoras de MMC

\section{Discussão}

A proposta deste estudo foi avaliar o impacto do nível de lesão funcional na função pulmonar de crianças portadoras de mielomeningocele. Foi observada presença de distúrbio ventilatório restritivo leve nas crianças com nível de lesão torácica e lombar-alta. E apesar de dentro dos limites da normalidade, as crianças com MMC com níveis inferiores de lesão apresentaram valores espirométricos menores que seus respectivos controles.

A literatura atual tem demonstrado que crianças com MMC apresentam elevada taxa de mortalidade $(1,2)$. Embora a falência renal seja a principal responsável por este achado, o distúrbio ventilatório-pulmonar é responsável por cerca de $25 \%$ dos casos de morte em crianças com MMC e, ao lado da disfunção cardíaca, é a principal causa de morte em adultos com MMC. Apesar desta estatística, a disfunção ventilatória ainda é um aspecto muitas vezes negligenciado na avaliação e acompanhamento desses doentes, principalmente quando criança (19).

Estudos sobre a manifestação pulmonar na MMC ainda são escassos e conflitantes. Dois diferentes estudos com crianças e adolescentes com MMC mostraram anormalidades do controle ventilatório $(8,20)$.

Nossos achados assemelham-se aos de Sherman et al. que observaram significante grau de doença restritiva pulmonar em adolescentes com MMC, além de evidenciarem também menor força muscular respiratória e capacidade de exercício de membros superiores comparados a controles saudáveis (5). Da mesma forma, o estudo de Swaminathan et al. evidenciou que em 5 dos 14 pacientes avaliados com MMC tinham evidência de doença pulmonar restritiva (8).

As manifestações neurológicas, ortopédicas, urológicas e gastrointestinais exteriorizam nos pacientes com MMC por limitação locomotora (cadeirantes), cifoescoliose, perda de força muscular, desnutrição ou obesidade. Estas, de forma isolada, afetam negativamente a função pulmonar, e, de forma conjunta, interagem exercendo maior impacto nessa função, especialmente nos pacientes com níveis de lesão funcional mais elevada.

A maioria das pessoas usuárias de cadeiras de rodas com mobilidade dependente adota um estilo de vida inativa que afeta diretamente a capacidade ventilatória-pulmonar, possivelmente, pelos baixos níveis de ventilação alveolar mantidos nesta condição. Estudos que envolveram pacientes tetraplégicos e paraplégicos mostraram que a função pulmonar está inversamente relacionada ao nível de lesão e à inatividade física destes pacientes $(21,22)$. Em paraplégicos, a média de $\mathrm{VEF}_{1}$ variou de $86 \%$ a $98 \%$ do previsto para a idade, sexo e altura, enquanto a CVF variou de $81 \%$ a $86 \%$ do previsto no caso dos eutróficos, e de $37 \%$ a $61 \%$ do previsto quando apresentavam baixo peso (18). Curiosamente, quando os cadeirantes vitimados de lesão medular foram submetidos a um programa de treinamento físico aeróbico com cadeira de rodas, um aumento expressivo foi observado nos índices de função pulmonar (23). Neste sentido, corroborando com nossos achados, pode-se inferir que a imobilidade subsequente à inatividade física dos portadores de MMC, cadeirantes, com nível de lesão torácica, sofre maior impacto na função pulmonar.

Ao lado do fator imobilidade, é bem conhecido que a cifoescoliose reduz a complacência pulmonar, e que se manifestada na primeira infância pode resultar em menor número de alvéolos configurando volumes pulmonares menores na vida adulta (24, 25). Em nosso estudo a criança com MMC com nível de lesão torácica, a despeito de ter realizado correção cirúrgica da cifoescoliose, apresentou valores reduzidos de CVF, sugerindo limitação ventilatória restritiva imposta pela deformidade da caixa-torácica. 
Adicionalmente, há evidências de que os extremos estados nutricionais (desnutrição e obesidade) exercem impacto na função pulmonar. Elevados IMC têm sido habitualmente associados à redução da $\mathrm{CVF}, \mathrm{VEF}_{1}$, capacidade pulmonar total, capacidade residual funcional, volume residual e função muscular respiratória em adultos $(26,27)$. Em crianças e pré-adolescentes estas associações ainda não são consistentes.

Por outro lado, há evidência de que crianças com desnutrição e atrofia apresentam menores valores de capacidade vital forçada e fluxos médios do que crianças com desnutrição sem atrofia e crianças eutróficas. A redução dos volumes e fluxos pulmonares nas crianças subnutridas pode ser explicada pela depleção muscular ventilatória. Porém, crianças subnutridas também apresentam comprometimento do crescimento esquelético, sendo esta mais uma razão para a função pulmonar reduzida $(28,29)$.

No presente estudo, podemos observar que todas as crianças com MMC apresentaram reduzido IMC com as curvas de percentis inferiores aos recomendados pela Organização Mundial de Saúde, sugerindo baixo estado nutricional quando comparado aos controles saudáveis (Tabela 1). Desta forma, podemos presumir que houve também a participação do fator nutricional na função pulmonar das crianças com MMC com níveis de lesão mais elevados, as quais o distúrbio ventilatório restritivo foi observado.

Logo, as hipóteses aqui levantadas devem ser cotejadas com dados obtidos de futuros estudos longitudinais. Com o protocolo utilizado, não foi possível avaliar o envolvimento dos fatores isolados que determinam a disfunção ventilatória, uma vez que certamente estes fatores se inter-relacionam nos portadores de MMC. Deve-se ressaltar, entretanto, que as crianças com MMC com níveis de lesão funcional torácica e lombar alta, as quais sofrem maiores consequências da imobilidade e cifoescoliose quando associadas a um baixo estado nutricional, apresentam menores índices de função pulmonar do que aquelas com relativa mobilidade preservada, dado os níveis de lesões mais baixas.

Algumas limitações podem ter ocorrido em nosso estudo. Primeiro, a amostra relativamente pequena de portadores de MMC. Segundo, não foi possível a avaliação da composição corporal para estimar a massa muscular, sendo, portanto, categorizada a desnutrição pelo IMC. Terceiro, a avaliação da força muscular respiratória não foi realizada. Quarto, embora não tenha sido este o objetivo, nenhum questionamento quanto ao nível de atividade física foi realizado, podendo certamente haver influência desta variável na função pulmonar. Quinto, diante do fato de não haver ainda literatura científica com valores de referência de normalidade de espirometria estabelecidos para a população brasileira, utilizamos os previstos de Polgar e Promadhat (18), porém também lançamos mão de um grupo controle pareado por idade e gênero.

As anormalidades pulmonares possivelmente são negligenciadas nas avaliações e seguimento dessa população, graças ao fato de a fraqueza muscular e a imobilidade mascararem os sintomas respiratórios, os quais podem não ser evidentes ao menos que haja uma situação em que o aumento ventilatório seja requerido.

Todavia, diante do fato de que crianças portadoras de MMC apresentam grande risco de se tornarem adultos com distúrbios respiratórios, e que todas as consequências da doença deterioram com a idade, especialmente aqueles com nível de lesão mais alta, faz-se necessário uma intervenção preventiva instituindo programas de condicionamento aeróbico, fisioterapia respiratória e orientação alimentar (30). Adicionalmente, consideramos cruciais avaliações longitudinais da função pulmonar, a fim de identificar precocemente anormalidades, e caso houver, prontamente oferecer a melhor estratégia terapêutica associando a fisioterapia respiratória à fisioterapia motora para os portadores de mielomeningocele.

\section{Conclusão}

Os achados do presente estudo mostram que crianças com mielomeningocele apresentam menores valores de função pulmonar quando comparados aos controles saudáveis, sobretudo os com níveis de lesão funcional elevada, os quais demonstraram distúrbio ventilatório restritivo. Adicionalmente, consideramos decisivo que a fisioterapia respiratória seja incorporada na avaliação e segmento das crianças com mielomeningocele, coadjuvante à fisioterapia motora, especialmente naquelas acometidas com níveis de lesões mais elevadas. 


\section{Referências}

1. Woodhouse CR. Myelomeningocele: neglected aspects. Pediatr Nephrol. 2008;23(8):1223-31.

2. Mitchell LE, Adzick NS, Melchionne J, Pasquariello OS, Sutton LN, Whitehead AS. Spina bifida. Lancet. 2004;364(9448):1885-95.

3. Baradarn N, Ahmadi H, Nejat F, El Khashad M, Mahdavi A. Nonneural congenital abnormalities concurring with myelomeningocele: report of 17 cases and review of current theories. Pediatr Neurosurg. 2008;44(5):353-259.

4. Schrander-Stumpel C, Fryns JP. Congenital hydrocephalus: nosology and guidelines for clinicalapproach and genetic counselling. Eur J Pediatr. 1998; 157(5):355-62.

5. Sherman MS, Kaplan JM, Effgen S, Campbell D, Dold F. Pulmonary dysfunction and reduced exercise capacity in patients with myelomeningocele. J Pediatr. 1997;131(3):413-8.

6. Waters KA, Forbes P, Morielli A, Hum C, O'Gorman $\mathrm{AM}$, Vernet 0 , et al. Sleep-disordered breathing in children with myelomeningocele. J Pediatr. 1998; 132(4):672-81.

7. Kirk VG, Morielli A, Gozal D, Marcus CL, Waters KA, D'Andrea LA, et al. Treatment of sleep-disordered breathing in children with myelomeningocele. Pediatr Pulmonol. 2000;30(6):445-52.

8. Swaminathan S, Paton JY, Ward SLD, Jacobs RA, Sargent CW, Keens TG. Abnormal control of ventilation in adolescents with myelodysplasia. J Pediatr. 1989;115(6):898-903.

9. Sarwark JF. Kyphosis deformity in myelomeningocele. Orthop Clin North Am. 1999;30(3):451-155.

10. Ronchi CF, Antunes LC, Fioretto JR. Respiratory muscular strength decrease in children with myelomeningocele. Spine. 2008; 33(3):73-75.

11. Zenteno D, Puppo H, González R, Vera R, Torres R, Chung-Yang K, et al. Evaluación de la musculatura de la inspiratoria en niños con antecedentes de mielomeningocele. Rev Chil Pediatr. 2008;79(1):21-25.

12. Guarnieri J, Vinchon M. Follow-up of adult patients with myelomeningocele. Neurochirurgie. 2008;54(5): 604-14.
13. Mitchell LE. Spina bifida research resource: study design and participant characteristics. Birth Defects Res A Clin Mol Teratol. 2008;82(10):684-91.

14. Buffart LM, van den Berg-Emons RJ, van Meeteren J, Stam HJ, Roebroeck ME. Lifestyle, participation, and health-related quality of life in adolescents and young adults with myelomeningocele. Dev Med Child Neurol. 2009;51(11):886-94.

15. Vinck A, Nijhuis-van der Sanden MW, Roeleveld NJ, Mullaart RA, Rotteveel JJ, Maassen BA. Motor profile and cognitive functioning in children with spina bifida. Eur J Paediatr Neurol. 2010;14(1):86-92.

16. Nihiser AJ, Lee SM, Wechsler H, McKenna M, Odom $\mathrm{E}$, Reinold C, et al. Body mass index measurement in schools. J Sch Health. 2007;77:651-671.

17. Sociedade Brasileira de Pneumologia e Tisiologia. Diretrizes para testes de função pulmonar. J Pneumol. 2002;28(supl 3):S44-S58.

18. Polgar G, Promadhat V. Pulmonary function testing in children: techniques and standarts. Philadhelphia: WB Saunders; 1971.

19. Singhal B, Mathew KM. Factors affecting mortality and morbidity in adults spina bifida. Eur J Ped Surg. 1999;9(Suppl 1):31-2.

20. Petersen MC, Wolraich M, Sherbondy A, Wagener J. Abnormalities in control of ventilation in newborn infants with myelomeningocele. J Pediatr. 1995; 126(6):1011-5.

21. Winslow C, Rozovsky J. Effect of spinal cord injury on the respiratory system. Am J Phys Med Rehabil. 2003;82(10):803-14.

22. Haisma JA, van der Woude LH, Stam HJ, Bergen MP, Sluis TA, Bussmann JB. Physical capacity in wheelchair-dependent persons with a spinal cord injury: a critical review of the literature. Spinal Cord. 2006; 44(11):642-52.

23. Le Foll-de Moro D, Tordi N, Lonsdorfer E, Lonsdorfer J. Ventilation efficiency and pulmonary function after a wheelchair interval-training program in subjects with recent spinal cord injury. Arch Phys Med Reabil. 2005;86(8):1582-6.

24. Jones RS, Kennedy JD, Hasham E, Owen R, Taylor JF. Mechanical inefficiency of the thoracic cage in scoliosis. Thorax. 1981;36:456-61. 
25. Berend N, Marlin GE. Arrest of alveolar multiplication in kyphoscoliosis. Pathology. 1979;11(3):485-91.

26. Clinical guidelines on the identification, evaluation, and treatment of overweight and obesisty in adults The Evidence Report. National Institutes of Healt. Obes Res. 1998;6(Supll 2):51S-209S.

27. Chinn DJ, Cotes JE, Reed JW. Longitudinal effects of change in body mass on measurements of ventilatory capacity. Thorax. 1996;51(7):699-704.

28. Nair RH, Kesavachandran C, Shashidar S. Spirometric impairments in undernourished children. Indian J Physiol Pharmacol. 1999;43(3):467-73.
29. Mansur SS, Neto FR. Desenvolvimento neuropsicomotor de lactentes desnutridos. Rev Bras Fisioter. 2006;10(2):185-91.

30. Brandão AD, Fujisawa DS, Cardoso JR. Características de crianças com mielomeningocele: implicações para a fisioterapia. Fisioter Mov. 2009;22(1):69-75.

Recebido: 14/12/2010

Received: $12 / 14 / 2010$

Aprovado: 18/03/2011

Approved: 03/18/2011 